\title{
Laboratory Research of the Two-Layer Liquid Dynamics at the Wind Surge in a Strait Canal
}

\author{
S.F. Dotsenko 1 , N.K. Shelkovnikov ${ }^{2, *}$ \\ ${ }^{1}$ Marine Hydrophysical Institute, Russian Academy of Sciences, Sevastopol, Russian Federation \\ ${ }^{2}$ M.V. Lomonosov Moscow State University, Moscow, Russian Federation \\ *e-mail: shelkovnikov@phys.msu.ru
}

\begin{abstract}
The results of laboratory experiments in a straight aerohydrocanal of the rectangular cross-section filled with the two-layer (fresh-salty) liquid are represented. The disturbance generator is the air flow directed to the area above the canal. The cases of the two-layer liquid dynamics in the canal with the horizontal flat bottom and in the presence of the bottom obstacle of finite width are considered. It is shown that during the surge in the straight canal, one of the possible exchange mechanisms on the boundary of fresh and salty layers may consist in the salt water emissions (resulted from the KelvinHelmholtz instability) to the upper freshwater layer. The subsequent eviction can possibly be accompanied by occurrence of undulations at the interface. Besides, the evictions can be followed by formation of the oscillating layer, i.e. the layer with maximum density gradient the oscillations of which propagate to the overlying layers.

Presence of the bottom obstacle complicates the structure of the two-layer liquid motions. In particular, it results in emergence of the mixed layers and transformation of the flow behind the obstacle into a turbulent one, formation of the wave-like disturbances over the obstacle, sharp change of the interface position and occurrence of large-scale vortices with the horizontal axes. It is revealed that the maximum peak of the flow velocity horizontal component is shifted upstream from the obstacle.
\end{abstract}

Keywords: waves in liquid, wind-induced processes, two-layer fluid, vertical exchange, flowing around of the bottom irregularities, laboratory simulation.

DOI: 10.22449/1573-160X-2017-3-3-18

(c) 2017, S.F. Dotsenko, N.K. Shelkovnikov

(C) 2017, Physical Oceanography

Inroduction. Development of the World Ocean is accompanied by the active use of its continental shelf. Although the shelf area is only $7.2 \%$ of the total area of the World Ocean, the share of mineral resources developed there is rather significant. Thus, $20 \%$ of the world's oil production and $15 \%$ of gas production are extracted from the sea bottom. Fishery, geological prospecting works and resource development are also carried out mainly in the shelf zone [1].

A distinctive feature of the water dynamics on the shelf is the wind-induced oscillations of the liquid surface, characterized by alternating elevation and sinking of the level at the opposite boundaries of the basin, depending on the wind direction. The spatio-temporal structure of these oscillations is determined by many factors, including the coastline shape, the bottom bathymetry slopes and its specific features, meteorological impacts, the geographic location of the shelf, its width and other characteristics that lead to complication of the processes occurring on the shelf and their forecast [2].

The wind-induced circulation promotes the redistribution of water masses not only in the horizontal direction, but also in the vertical one, which causes the exchange of coastal waters with the open part of the World Ocean. An important manifestation of the wind-induced motions is the displacement of the seasonal pycnocline on the sea shelf. Depending on the vertical circulation intensity, 
the structure and depth of the seasonal thermocline change. This leads to an increase or decrease of the upper quasi-homogeneous layer thickness.

The results of theoretical studies and instrumental observation data point to the fact that with the development of gradient and drift currents, the evolution of low-frequency surface and internal waves in regions with abrupt changes in the bottom topography, the phenomena typical only for these regions are possible. Particularly, such phenomena include transformation, capture, reflection from the bottom irregularities and wave scattering, intense vertical movements of water masses, sharp changes in the depth of seasonal pycnocline occurrence, etc. [3, 4]. The study of these processes in the in situ conditions requires special organization of measurements, which is carried out by means of laboratory experiments $[5,6]$.

The aim of the present article is an experimental (laboratory) study of dynamic processes in a canal filled with a stratified liquid. In the experiments described below, special attention is paid to research of the exchange mechanisms between the layers of a two-layer liquid.

Laboratory facility scheme. The experiments were carried out in a $15 \times 15 \times$ 280 (330) cm straight aerohydrocanal (Fig. 1). Transparent walls made it possible to observe the process of wave propagation along the canal and to take video and photos. The air flow was created by a fan that pumped air between the water surface and the canal lid through a special box. Changing the tilt of this box allowed setting the desired angle of air flow entry into the canal. The fan was connected to the box using flexible corrugated aluminum hoses. The fan was equipped by the unit to regulate the air flow through the sleeves into the canal. Due to this, it was possible to change the wind speed at the outlet from the box from 3 to $10 \mathrm{~m} \cdot \mathrm{s}^{-1}$. The airflow velocity profile in the canal was measured at different velocities in the canal inlet using a digital anemometer with an external impeller.

Measurement of the salinity distribution was carried out by an electrical conductivity sensor. Its position is diagrammatically shown in Fig. 1. The sensor consisted of two steel rods with a diameter of $1 \mathrm{~mm}$ and a length of $10 \mathrm{~cm}$, located at a $5 \mathrm{~mm}$ distance each other. The rods were insulated. There were building-ups made of $1 \mathrm{~mm}$ platinum wire with their ends facing each other in their lower part. Only these two end platforms had electric contact with water, the gap between them was $2 \mathrm{~mm}$.

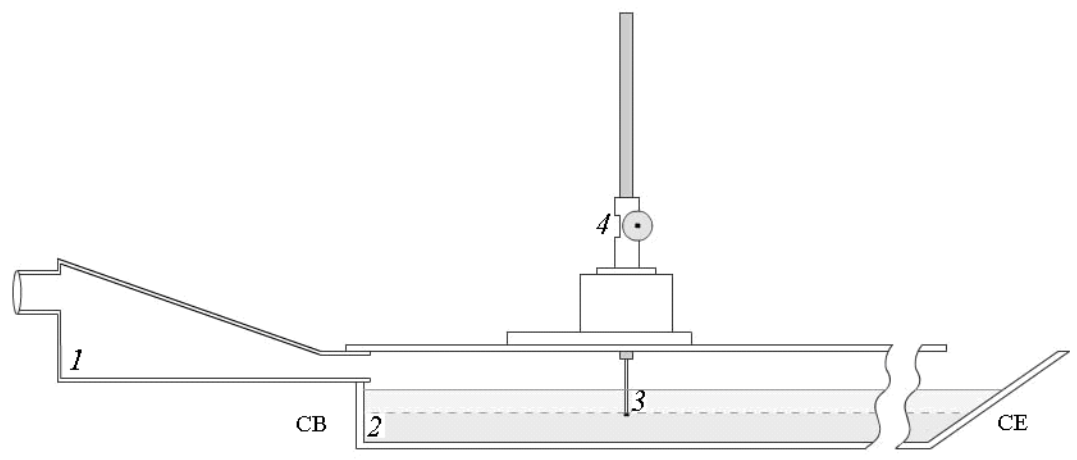

Fig. 1. Laboratory facility scheme: 1 - box; 2 - $15 \times 15 \times 350 \mathrm{~cm}$ aerohydrocanal; 3 - salinity sensor; 4 - micrometer scale; $\mathrm{CB}$ - the canal beginning; $\mathrm{CE}-$ the canal end 
The conductometric method was used to measure the salinity (salinity measurement by the electrical conductivity of the saline solution). The sensor was connected to the alternating current circuit, and then the amplitude modulated signal was supplied through the interface unit to the ADC computer board. The sensor entering in a higher salinity layer resulted in the electrical resistance decrease between the sensor platinum contacts and, as a consequence, in a decrease in the voltage drop at the ADC board input. The sensor calibration was carried out by an absolute method. It allowed to obtain the voltage drop variations at the board input depending on the concentration of the saline solution. The calibration was first carried out in tap water, then in the solution with the salinity increased to $20 \%$ with $2 \%$ step. The accuracy of preparation of saline solutions during calibration was about 3\%.

Generally, the dependence of the $R$ liquid electrical conductivity on depth is a nonlinear function. Therefore, the measuring system introduces some nonlinear distortions into the measured signal. The nonlinear dependence of the voltage on the immersion depth of the salinity sensor was approximated by means of the $4^{\text {th }}$ degree polynomial. The approximation error magnitude did not exceed 5\%. Salinity profiles were removed after flow attenuation in the laboratory canal after completion of each cycle of air flow exposure to the liquid. The procedure described above makes it possible to measure variations in the upper quasi-homogeneous layer thickness and the density jump.

The semicircular cylinders with base radii of $r=1.3 \mathrm{~cm}$ and $r=2.5 \mathrm{~cm}$ placed horizontally on the bottom were used in the experiments on the effect of bottom unevenness on the processes in a two-layer liquid. The cylinders were set at a distance of $80 \mathrm{~cm}$ from the beginning of the CB canal (the air flow supply place, see Fig. 1).

The main source of dynamic process data was video recording. The drift velocity on the surface and inside the liquid was measured by observing the motion of polystyrene balls with neutral buoyancy, which, depending on their size, were distributed throughout the depth of the liquid. The accuracy of the measurements was about $10 \%$. Video recording made it possible to obtain high-quality videos, which were subsequently used to determine the quantitative features of the observed processes.

The two-layer liquid dynamics in the case of a horizontal bottom. Experimental studies of the features of dynamic processes taking place under the surge wind effect in a rectilinear canal with a flat bottom were carried out both with a bottom obstacle taking into account its effect on the stratified liquid motion and without it.

A series of observations of a two-layer liquid was conducted under various experimental conditions, including changes of the canal length, obstacle dimensions and wind speed ranging from $3.5 \mathrm{~m} \cdot \mathrm{s}^{-1}$ to $10 \mathrm{~m} \cdot \mathrm{s}^{-1}$.

The canal was first filled with salt, then with fresh water. To prevent mixing with salt water, the fresh water was poured using a system of flexible thin hoses attached to an inclined surface with a paper sheet was laid on it. The outflowing water moved smoothly from it to the salt water surface. The floating paper sheet rose as the channel filled. It resulted in a density structure formation in the canal, consisting of two quasi-homogeneous layers (the upper fresh layer and the lower saline one) and the transition layer between them. The total depth of canal filling was $8 \mathrm{~cm}$. The initial thickness of the salt layer (20\%) was $4 \mathrm{~cm}$. After filling the canal, a fan was activated. It was pumping the air flow into the space between the water surface and the canal lid. The wind effect on the water surface had lasted for 
a predetermined time period. Then the fan was turned off. Further, using a conductivity sensor, the vertical distribution of salinity was measured.

During the laboratory experiment, from the moment the fan was turned on and until the flows in the liquid were attenuated, the processes occurring in the channel were recorded by a video camera in a visual window $10 \mathrm{~cm}$ wide at a $80 \mathrm{~cm}$ distance from the canal beginning.

Under the wind flow effect, fresh water surge was created in the canal end, opposite from the wind entrance point. The drift current, being a result of the surge, caused a compensatory countercurrent. As a consequence, the boundary between the layers at the end of the CE canal was decreased (see Fig. 1) and increased in the beginning of the $\mathrm{CB}$ canal.
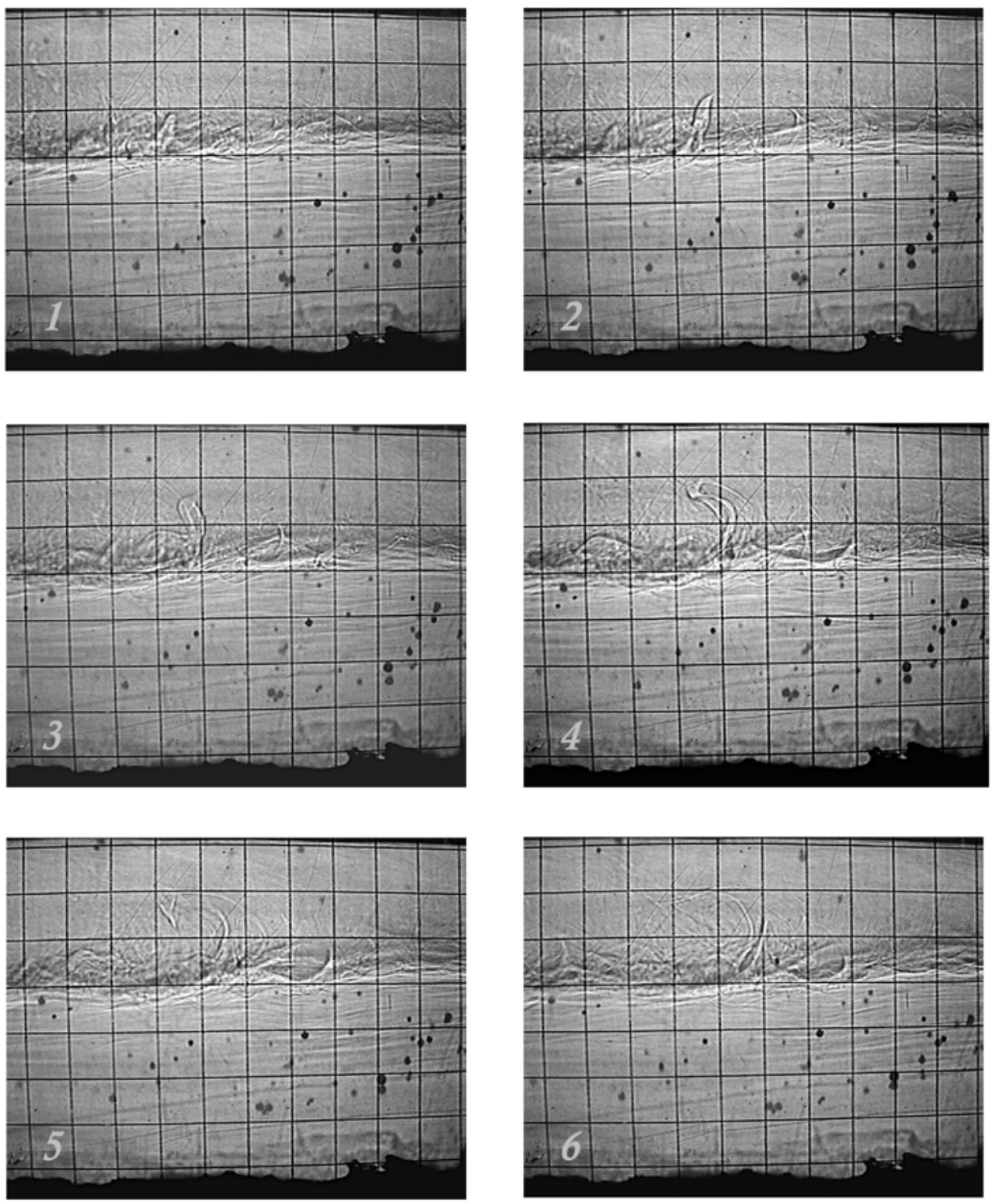

Fig. 2. The process of formation and further development of a "protuberance". The time interval between consecutive pictures is $0.4 \mathrm{~s}$ 
During the wind impact, the appearance of "protuberances", i.e. the water discharge from the lower saline to the upper fresh water layer, was observed (Fig. 2). This process occurs as a result of the Kelvin - Helmholtz instability and is characterized by an increase in the amplitude of wave-like oscillations. According to [7], in the stratified current mixing layer, the current velocity over the crest and under the hollow is higher than under the crest and above the hollow. Since the pressure in lower velocity areas is higher, the reduced pressure zones are formed directly above the crest tops, being accompanied by the formation of emissions.

Thus, the formation of "protuberances" is one of the possible mechanisms for energy exchange between the layers both in the upward and downward direction. This process eventually leads to salinization of the upper liquid layer and an increase in the thickness of the upper quasi-homogeneous layer. The existence of vertical exchange due to the formation of "protuberances" is confirmed in experiments and is clearly visible in Fig. 2.

According to the data of the conducted experiments, the lifetime of a protuberance is about $2 \mathrm{~s}$ under the surge wind, the horizontal velocity of its propagation is $0.5 \mathrm{~cm} \cdot \mathrm{s}^{-1}$ and the height of the liquid kick is $2.5 \mathrm{~cm}$, which is about $30 \%$ of the total depth of the entire layer of the liquid.

During the wind exposure, three layers with different salinity distributions are formed: a top layer with almost fresh water, a transition layer with a large salinity gradient and a bottom layer with unmixed salt water.

After the wind exposure termination, the return currents caused by fresh and salt water surges are formed. In such conditions, the velocity field in the layers moving in different directions differs from the considered above structure of currents formed by the wind impact. The difference lies in the fact that under the absence of wind, the density jump and current velocity layer is twice as thick as when exposed to wind. As a result, any perturbation formed in the jump layer was twisted in the direction of the current on the surface of the liquid.
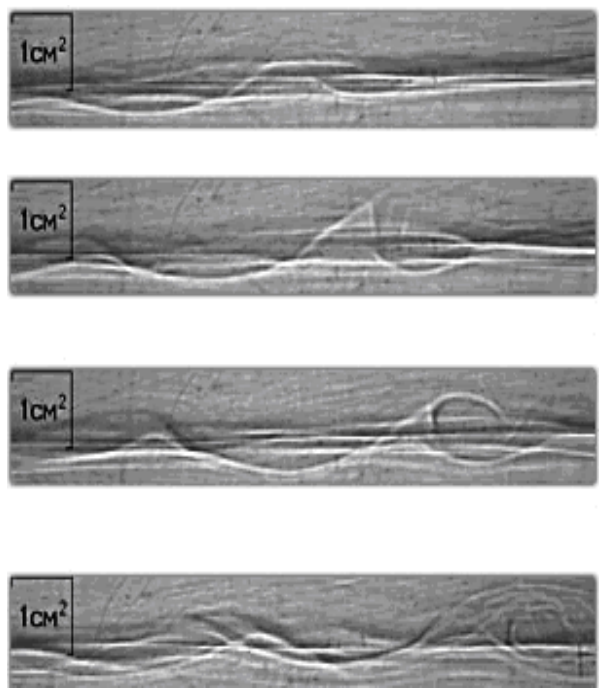

$a$

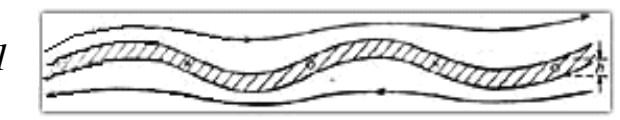

2

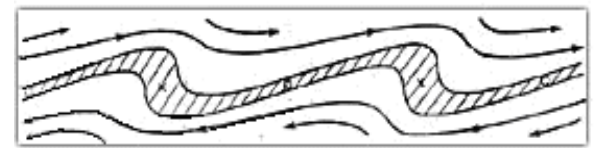

3
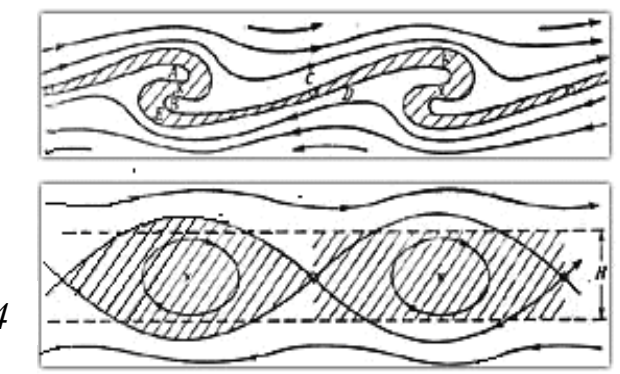

$b$

Fig. 3. Formation of a "protuberance" after the wind termination: $a$ - experimental results (the time step between the pictures $1-3$ is $1.2 \mathrm{~s}$, between the pictures 3 and 4-2.8 s); $b$ - numerical simulation [7]

PHYSICAL OCEANOGRAPHY NO.3 (2017) 
According to [7], the following stages of instability development are observed in the mixing layer of the stratified current (Fig. 3):

- formation of sinusoidal waves at the two-layer liquid interface, the so-called oscillations of the vortex layer;

- increase of the steepness of wave-like motions at the interface caused by the expansion of the vortex layer in the ascending nodes and the vorticity increase in the descending nodes;

- vortex twisting with the formation of a "roll" with rotation. This tendency is especially pronounced under the small density difference and large velocity shifts. At this stage, the liquid appears at the $a$ point. It has a higher density than at the $b$ point, and, consequently, the liquid is in an unstable state and starts to mix in the coiled vortices. If there is a significant difference in densities of the layers, the mixing begins immediately after the second stage of the instability development;

- formation of a structure where the mixed liquid forms such instabilities as "cat's eyes". At that the wave motion continues to develop in the overlying and underlying layers.

Comparison of the experimental data (Fig. 3, a) with the results of computational experiments (Fig. 3, b) [7] showed that the nature of the disturbances at the interface between the layers is qualitatively the same.

As previously mentioned, after the wind exposure termination, the reverse currents are formed. At the same time the fresh layer moves to the canal beginning, and the salt layer moves to the canal end. In the case of a light wind, the layer thickness variations at opposite ends of the canal (such as seiche oscillations) are observed. Under a relatively strong wind, the salt layer moves to the canal end in a way that all the salt water concentrates in half of the canal opposite the fan (Fig. 4). At that, the salt layer position at the canal beginning occupies the transition layer. When the salt water mass at the canal end reaches a certain value, the liquid motion direction changes to the opposite one. As a result, a part of the salt layer, propagating to the canal beginning, meets its back front, which moves toward the canal end. After the collision, perturbations are formed at the interface between the salt and transition layers and a wave-like motion of the density jump is observed.

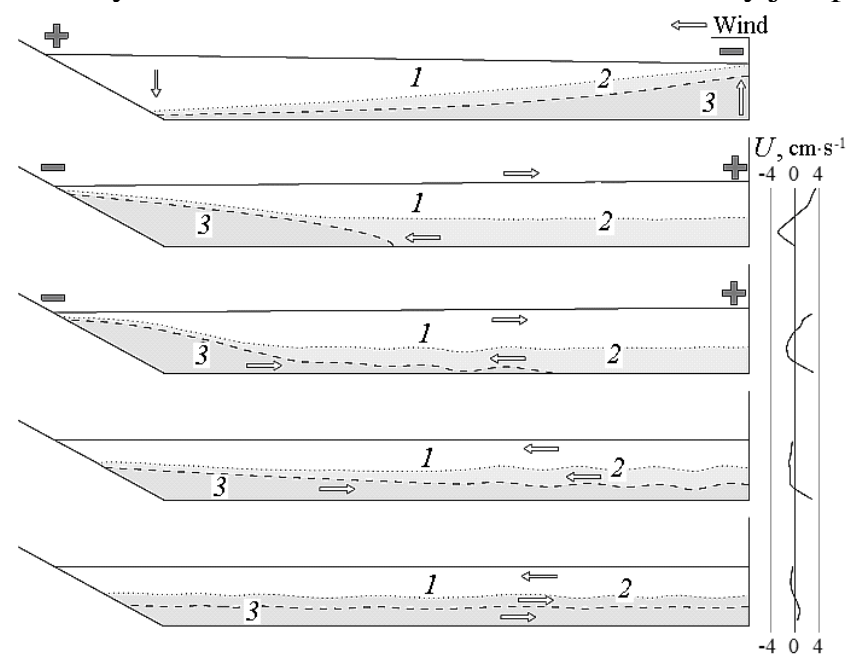

Fig. 4. The development of oscillations in the near-bottom layer of a two-layer liquid in a canal with a horizontal bottom after the wind termination: 1 - a fresh water layer; 2 - an intermediate layer; 3 a salt water layer 

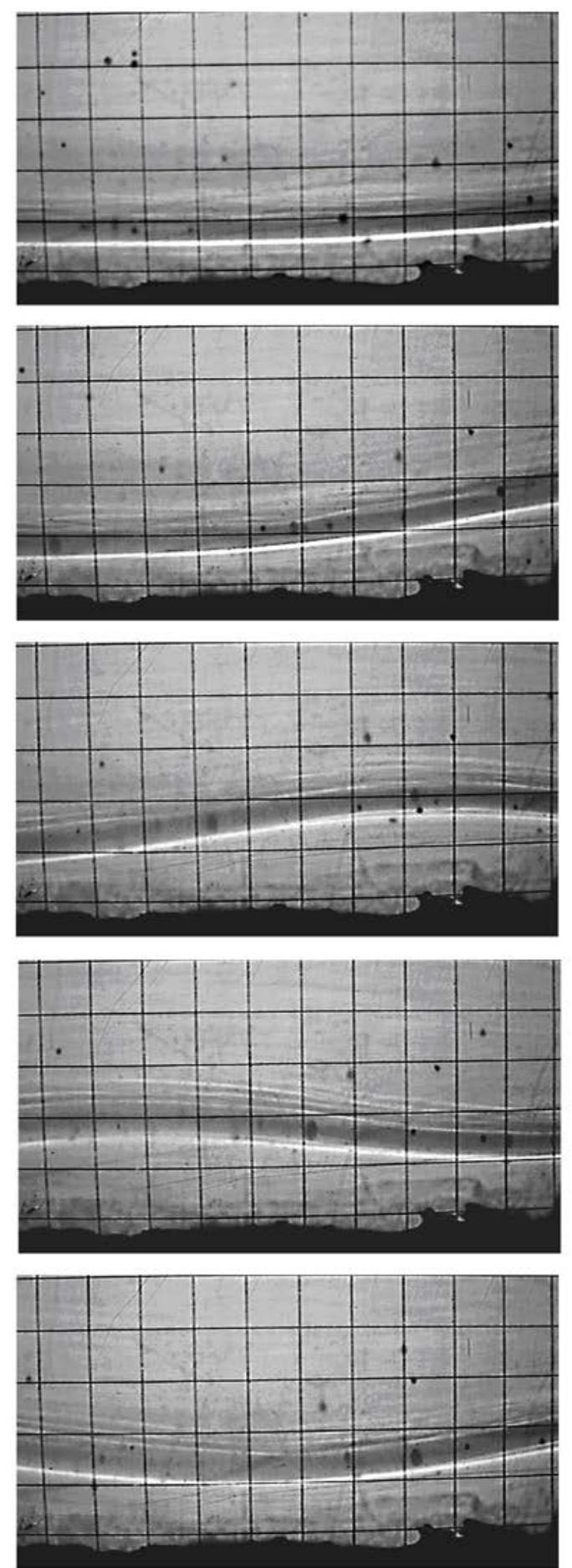

$\lceil 1 \mathrm{~cm}$

Fig. 5. The stratified layer oscillations after the wind termination. The time step between the pictures is $2 \mathrm{~s}$

PHYSICAL OCEANOGRAPHY NO.3 (2017) 
Spreading along the bottom, the salt layer displaces the lighter transition layer upward and propagates to the hydrocanal beginning. Reflecting from the canal beginning, the oscillating layer of the maximum salinity gradient is observed. Its oscillations propagate into the upper layers. Amplitude of the oscillations of the layers located above decreases moving from the underlying layer, so that the surface does not experience perturbations. Owing to the continued flow of salt water into the canal, the oscillating layer rises from the bottom to the middle of the canal. During 2 minutes, the oscillations at the boundary of these layers are fading out gradually. The layer oscillation process is shown in Fig. 5.
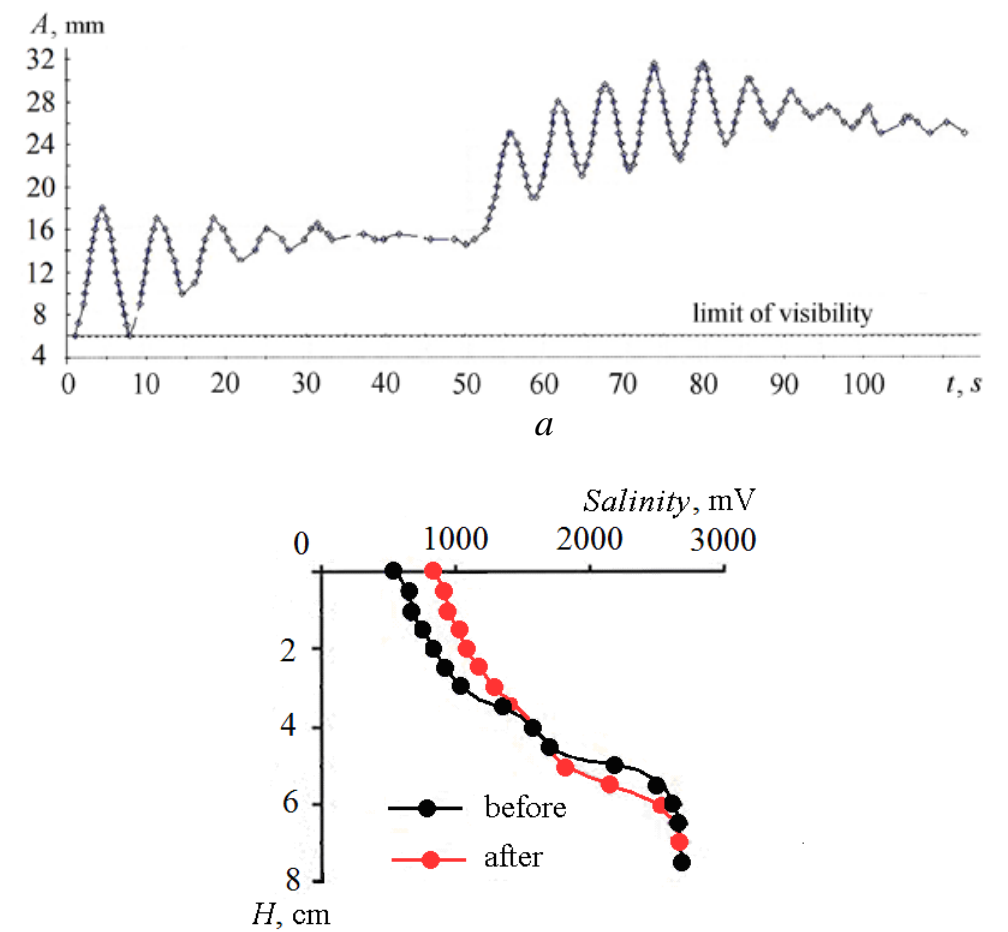

$b$

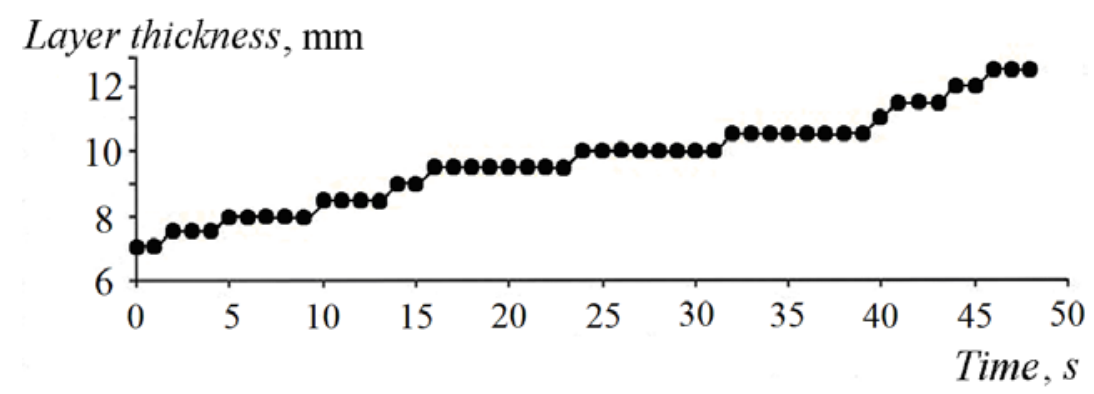

C

Fig. 6. Features of the oscillations of the upper layer of the liquid: $a$ - time variation of the position of the upper boundary of the salt liquid layer; $b$ - vertical distributions of the salinity before the wind exposure beginning and after its termination; $c$-time variation of the occurrence depth of the upper boundary of the salt liquid layer 
The time variation nature of the position of the upper boundary of the salt layer is shown in Fig. 6, $a$. During the first 40 seconds (after the oscillating layer occurrence in the video recording window), there is a propagation of oscillations to the canal beginning, where they are reflected from the vertical wall and change the direction of motion. At the 55th second, the propagation of the layer oscillations starts at the canal end. Insomuch as the salt water inflow goes on in the canal beginning, this causes a rise in the interface.

According to Fig, 6, $a$, the time interval between the wave crests is $6 \mathrm{~s}$, the amplitude of the oscillations reaches $1.2 \mathrm{~cm}$, the height of the oscillating layer rise is $2.5 \mathrm{~cm}$, the mean velocity is $3-5 \mathrm{~cm} \cdot \mathrm{s}^{-1}$.

Due to the fact that the mixed water continues to flow to the canal beginning, the oscillating layer thickening is observed (Fig. 6, $c$ ).

Before and after the experiment, the salinity distribution was measured: the thickness of the liquid transitional layer increased from $0.7 \mathrm{~cm}$ to $1.2 \mathrm{~cm}$ (Fig. 6, b). Owing to a strong air flow (about $10 \mathrm{~m} \cdot \mathrm{s}^{-1}$ ) during the experiment the water was strongly mixed. The salt layer, although it became thinner, did not mix with the overlying layers of liquid.

The spatial pattern of the oscillating layer is shown in Fig. 7. The data is taken from an experiment which involved the shooting at the canal beginning. The oscillating layer is shown after the reflection of wave-like disturbances from the vertical wall at the interface between the salt water layer and the transition one. The distance between the crest peaks was about $20 \mathrm{~cm}$.

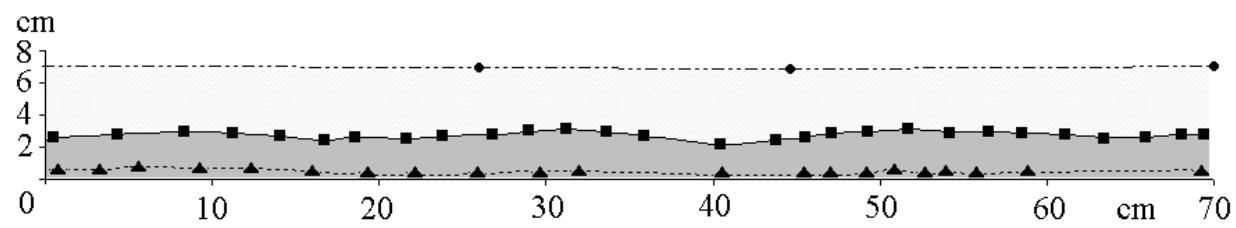

Fig. 7. The liquid surface position $(\bullet)$, interfaces of the layers $(\square)$ and basin bottom ( $\boldsymbol{\Delta}$ )

Therefore, under the surge wind, the "protuberances" are formed, causing a vertical exchange between the liquid layers of a different density, the wave-like motions, going through all the stages of instability development described in [7] at the subsequent time instants, and finally the so-called oscillating layer appears.

Influence of the bottom unevenness on the stratified liquid dynamics. To investigate the bottom unevenness influence on the stratified liquid flow the experiments at various air velocities with bottom obstacles in the form of a semicircular cylinder were carried out. The small section obstacle had a radius equal to $1 / 3$ of the salt layer thickness, the large section one $-2 / 3$ of its thickness.

Fig. 8 shows the distribution of the horizontal component of the velocity under the large obstacle flowing around by salt water, depending on the distance to the top of the obstacle $(r=2.5 \mathrm{~cm})$. The flow velocity was measured at a height of not more than $1 \mathrm{~cm}$ above the obstacle after turning off the fan, when the return flows were formed. 


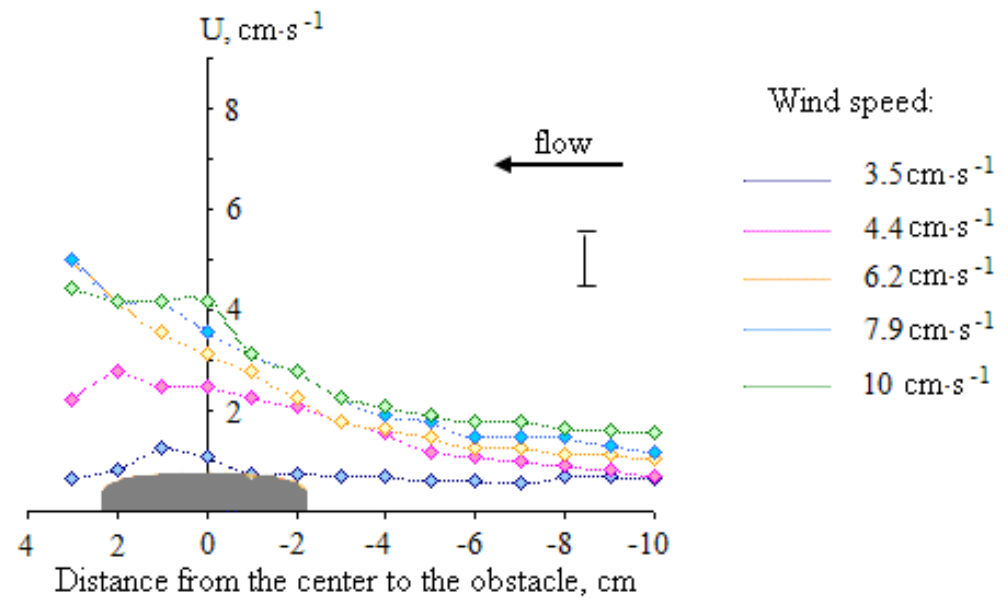

Fig. 8. Distribution of the horizontal velocity of the salt water flow at the height of not more than $1 \mathrm{~cm}$ over an extended obstacle after the wind exposure termination and the return flow formation

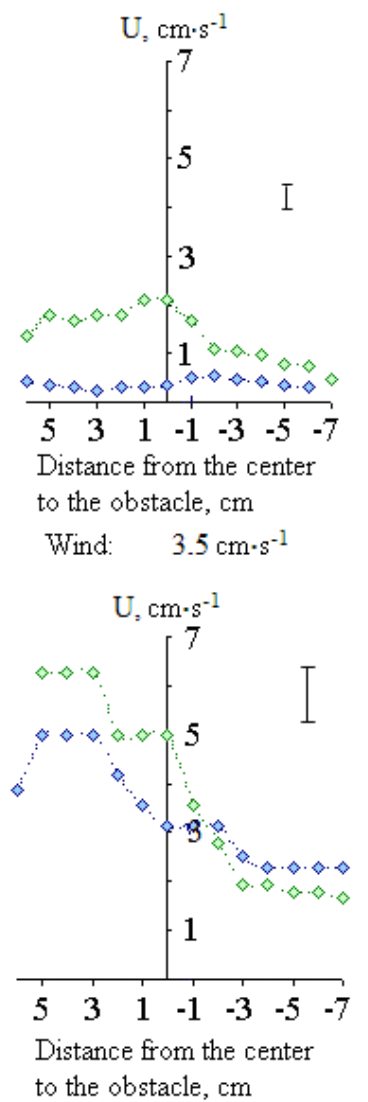

$7.9 \mathrm{~cm} \cdot \mathrm{s}^{-1}$

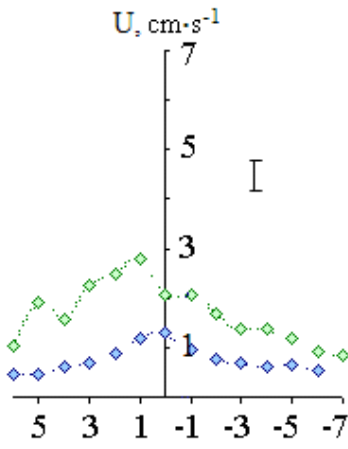

Distance from the center

to the obstacle, $\mathrm{cm}$

$4.4 \mathrm{~cm} \cdot \mathrm{s}^{-1}$

$\mathrm{U}, \mathrm{cm} \cdot \mathrm{s}^{-1}$

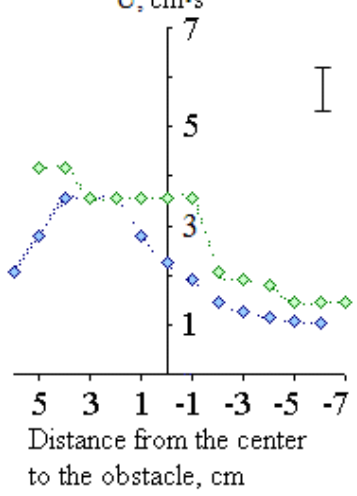

$10 \mathrm{~cm} \cdot \mathrm{s}^{-1}$

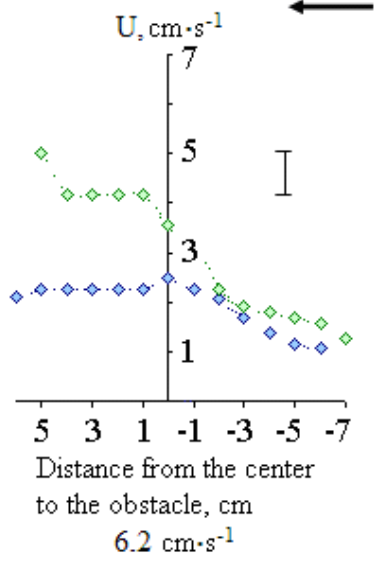

$6.2 \mathrm{~cm} \cdot \mathrm{s}^{-1}$

\section{Радиус \\ препятствия:}

$\diamond-1.3 \mathrm{~cm}$

$\diamond-2.5 \mathrm{~cm}$

Fig. 9. Horizontal velocity distributions along the horizontal coordinate for obstacles of different cross-sections, depending on wind speed and bottom obstacle radius 
A comparison of the vertical velocity profiles (horizontal component) under the flowing around by small and large obstacles is shown in Fig. 9. Velocity of the large obstacle flowing around can exceed several times the velocity of the small obstacle flowing around. Thus, under the light wind $\left(3-4 \mathrm{~cm} \cdot \mathrm{s}^{-1}\right)$ the velocity over a large obstacle could be $2-3$ and sometimes 6 times more than the velocity of the flow over a small obstacle. Under a strong wind $\left(7-10 \mathrm{~m} \cdot \mathrm{s}^{-1}\right)$, the ratio of velocities became up to 1.5 times less.

Under the light wind exposure $\left(4-5 \mathrm{~m} \cdot \mathrm{s}^{-1}\right)$ on the leeward side of the obstacle, a mixing layer was formed (Fig. 10) being a result of the Kelvin-Helmholtz instability development. It was revealed that at the initial stage the mixing layer thickness increases linearly with increasing of the distance from the top of the obstacle. Then the mixing layer reaches the bottom, and the flow passes into the bottom turbulent stream. The mixing layer thickness increases with time. Such a type of the obstacle flowing around can be compared with the mixing layer development model [8] for an inclined bottom in a two-layer flow of mixing liquids.

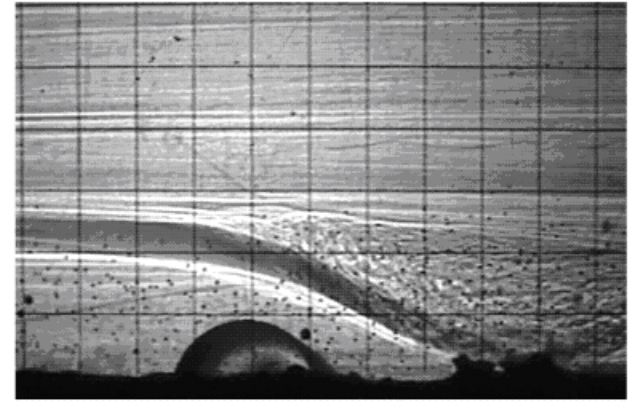

(a)

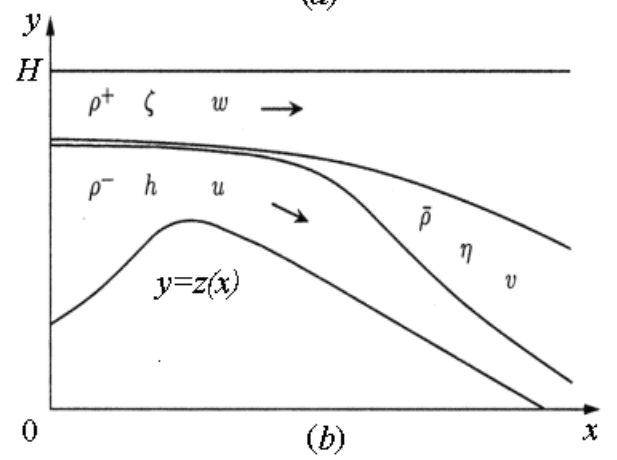

Fig. 10. The mixing layer $(a)$ in the case the obstacle flowing around by a stratified flow $(r=1.4 \mathrm{~cm}$, wind speed $-4 \mathrm{~m} \cdot \mathrm{s}^{-1}$ ) and the scheme of its formation $(b)$ according to the model [9]

When the experiments were carried out in the canal with a large semicylinder, the undulating perturbations followed the obstacle at the bottom. At the same time the height of the crests was approximately equal to its height (Fig. 11, a). Depending on the wind speed, one or two wave crests may appear. Such disturbances are due to the fact that in the presence of a bottom obstacle with a radius $r=2.5 \mathrm{~cm}$, being almost $1 / 3$ of the entire liquid depth, "blocking" of the liquid along the flow upward of it takes place, i.e. the semicylinder prevents further spreading of the near-bottom flow, having thickness of less than the height of this 
semicylinder. When the height of the salt water surge is insignificantly higher than the height of the obstacle, after the wind exposure termination in the opposite direction from the canal beginning, only the mixed water of the transition layer and the upper part of the saline liquid are moving. The resulting flow tends to occupy the lowest position in the course of flowing around, but collides with a salt layer that does not move due to the "blocking" of the return near-bottom flow of salt water on the obstacle. Since this flow, having reflected from the vertical wall of the canal end, changed its direction, as a result of the salt water inflow, these disturbances were moving to the obstacle which they were eroded on. It can be noted that the process of "blocking" becomes important in very slow currents or in the presence of obstacles of great height.

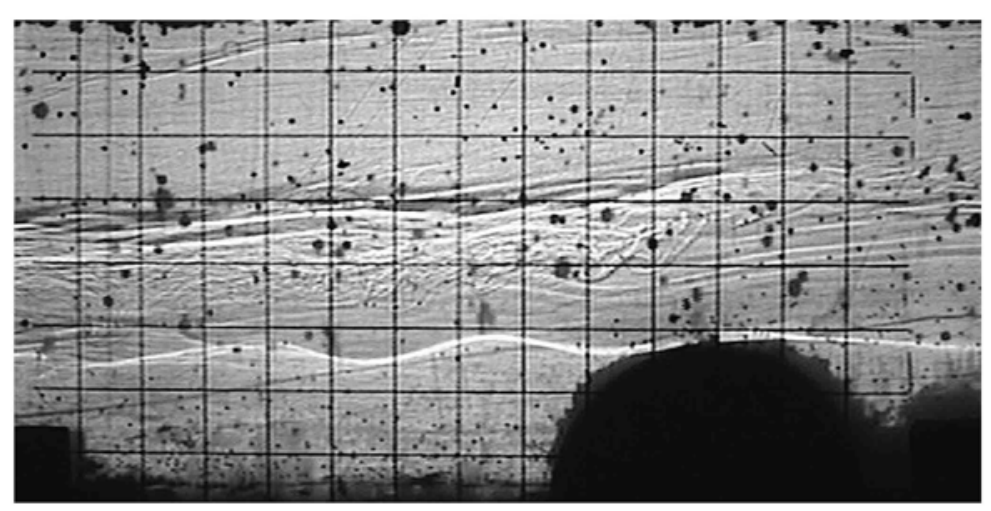

$a$

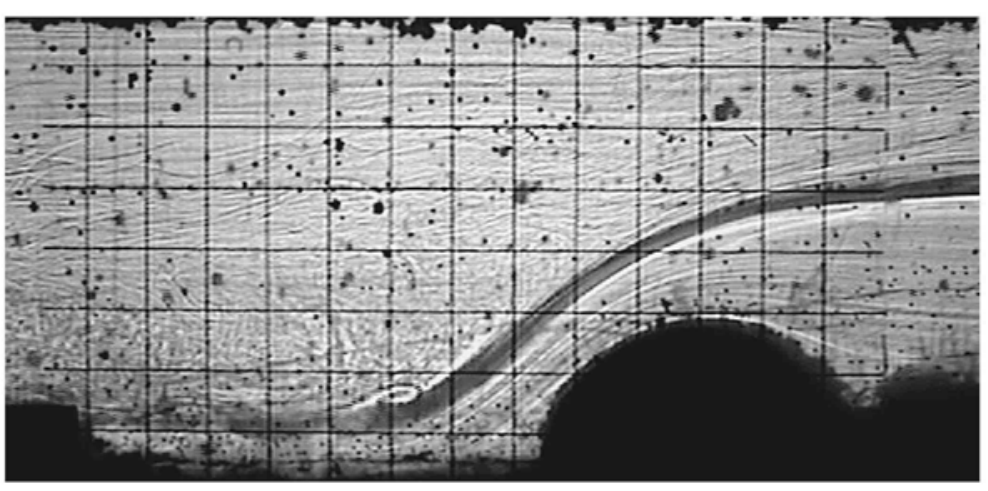

$b$

Fig. 11. The formation of wave-like disturbances behind an obstacle of radius $r=2,5 \mathrm{~cm}(a)$ and near-wall flow under the obstacle flowing around at a wind speed of $8 \mathrm{~m} \cdot \mathrm{s}^{-1}(b)$

During the relatively strong wind exposure on the liquid the pattern significantly changes. The heavier liquid always tends to occupy the lowest position on the leeward side of the obstacle. In our experiments with a strong wind, when the salt water surge at the canal beginning is quite large and significantly exceeds the height of the obstacle, the powerful slope flows are formed (Fig. 11, b). 


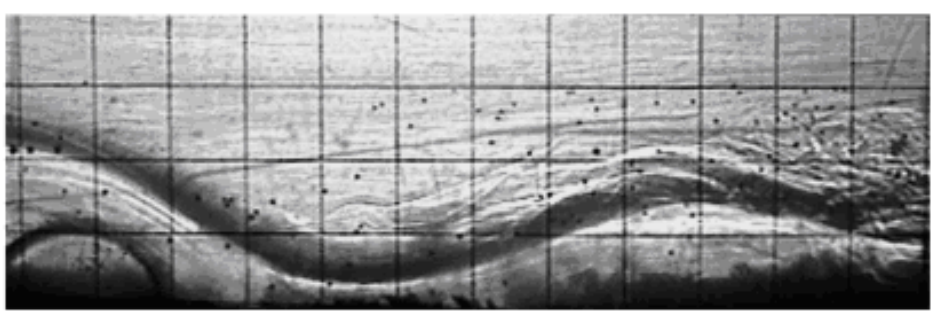

$a$

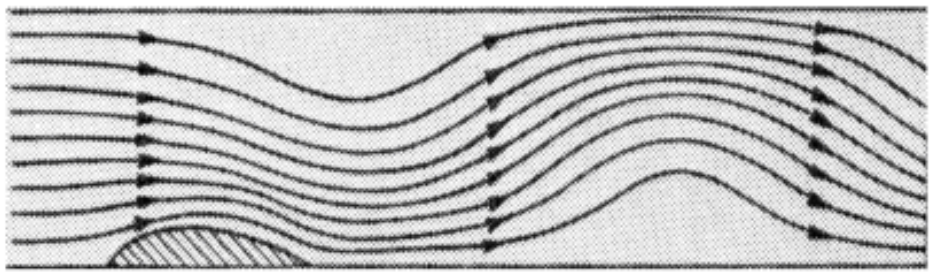

$b$

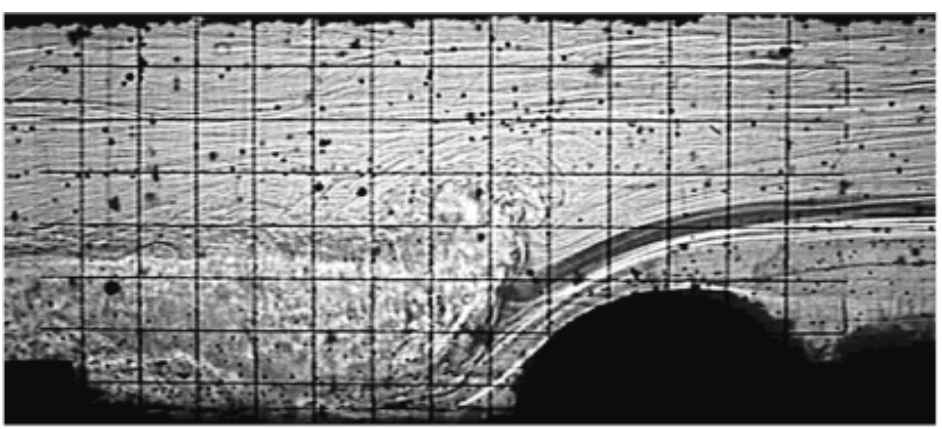

$C$

Fig. 12. The obstacle flowing around by a stratified liquid flow: $a$ - experimental results $(r=1.3 \mathrm{~cm}$, wind speed $-8 \mathrm{~m} \cdot \mathrm{s}^{-1}$ ); $b$ - the calculation in accordance to the work [9]; $\mathrm{c}$ - the flow structure behind the obstacle and the mixing layer formation $\left(r=2.5 \mathrm{~cm}\right.$, wind speed $\left.-6 \mathrm{~m} \cdot \mathrm{s}^{-1}\right)$

Under a strong wind (about $8 \mathrm{~m} \cdot \mathrm{s}^{-1}$ ), the flowing around of a small obstacle by a stratified flow was accompanied by a change in the pycnocline position. So (under the incomplete mixing) immediately after the obstacle, the interface was buried to $0.2 \mathrm{~cm}$ and then rose to $1.4 \mathrm{~cm}$, forming a "hump" (Fig. 12, a), which existed for 30 seconds. The "hump" formed by the salt water flow was destroyed as it was turbulized by the salt water reflected from the vertical wall of the canal beginning. Similar oscillatory motions were observed in [9] in the study of the deformations of liquid lines of stratified flow after passage of the barrier. A comparison of the lines calculated in [9] for obstacles of different shapes and heights with flow structures observed in the series of laboratory experiments described above was carried out. Although these oscillatory motions obtained to be weaker than those calculated, nevertheless, the correspondence between theory and experiment was noted. 


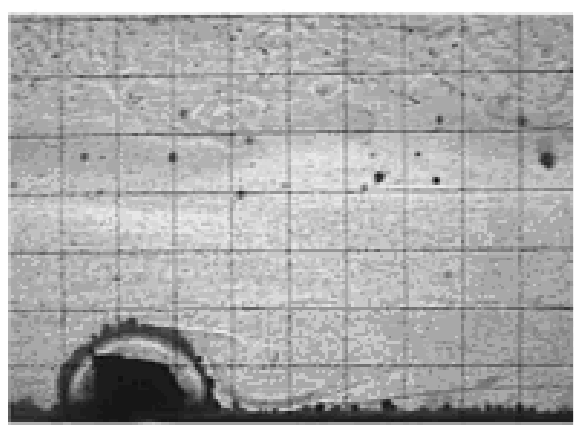

$a$

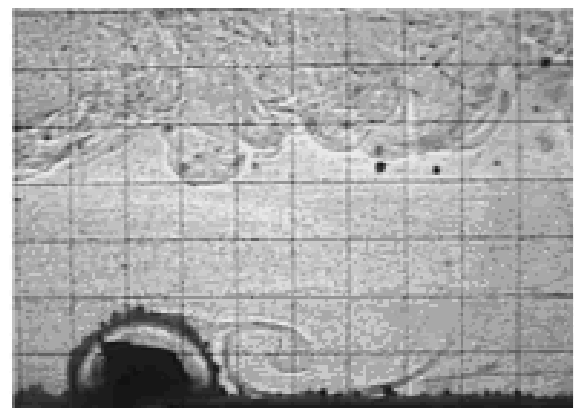

$C$

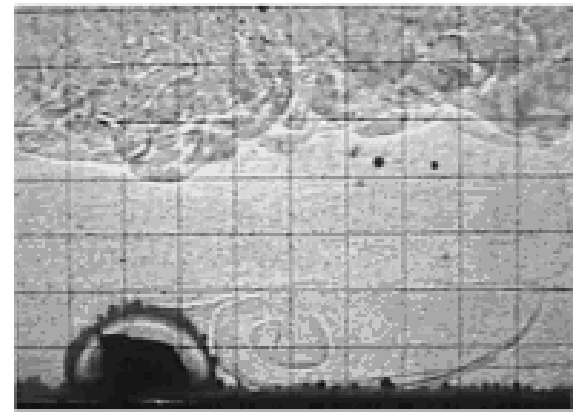

e

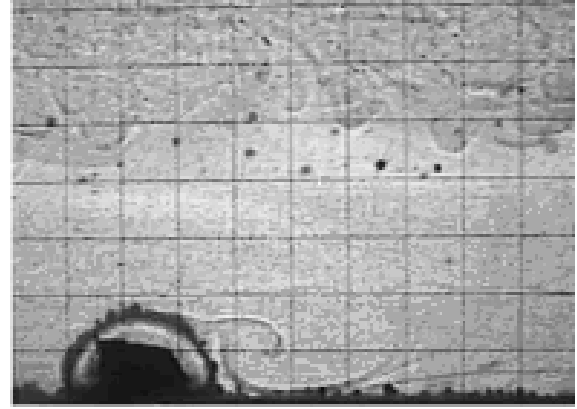

$b$

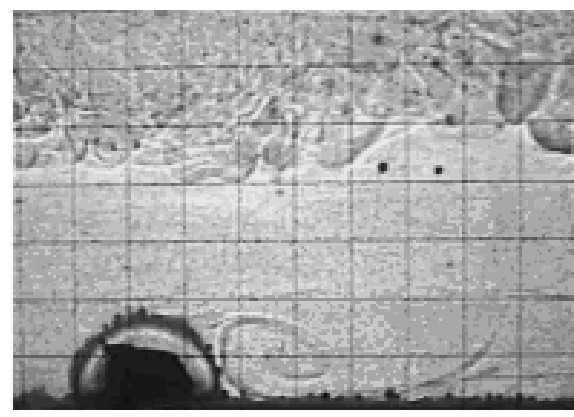

$d$

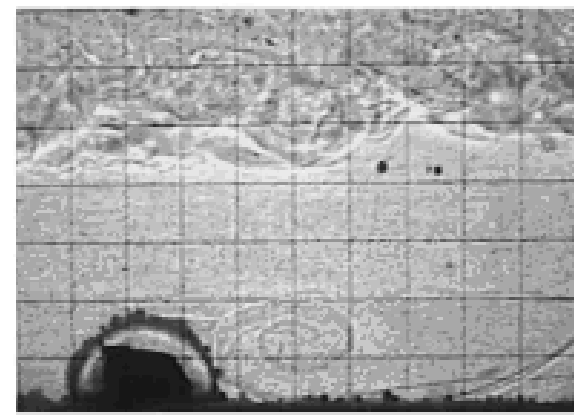

$f$

Fig. 13. Formation of a vortex with a horizontal axis under flow around the bottom obstacle of radius $r=1.3 \mathrm{~cm}$. Six video shots $(a-f)$ with a time interval of $1.25 \mathrm{~s}$ are given

Under a large obstacle flowing around, the flow velocity exceeded the flow velocity of the small one, the upper boundary of the salt layer lost its stability, and as a result the salt water flow was turbulized. At the same time a mixing layer was formed on the leeward side of the obstacle. This layer was connected with the turbulent flow, passing into it. In experiments with a small near-bottom obstacle on its leeward side, a large vortex with a horizontal axis was formed and developed as a result of the separation of the boundary layer from the obstacle (Fig. 13). The vortex appeared in the first seconds of the wind exposure, when the compensatory countercurrent was only starting up.

Blow the possible effect of the bottom unevenness on the processes observed in the even bottom canal is considered. 
The obstacle has no effect on the instability formation at the interface, i.e. the formation of "protuberances". Perturbations, appeared at the interface between salt water and the transition layer, were "blocked" upward along the flow from the obstacle, so that the oscillating layer was not observed.

As is seen from the foregoing, the pattern of the observed processes becomes more complicated in the presence of the bottom unevenness. It was revealed that the presence of an obstacle promotes the formation of a mixing layer, the appearance of wave perturbations, the deformation of the pycnocline near the obstacle and the formation of large vortices with a horizontal axis. Therefore, the presence of bottom surface unevenness significantly affects the behavior of the stratified liquid.

Conclusions. The following conclusions can be made based on the laboratory experiments carried out.

In the rectilinear canal under the surge one of the possible mechanisms of exchange at the boundary between the fresh and salt layers directed in different sides can be the so-called "protuberances", i.e. the discharge of salt water into the upper fresh water layer. They are a result of the Kelvin-Helmholtz instability. In the subsequent surge, the wave-like motions may appear at the boundary of the layers. Their evolution stages satisfactorily agree with the instability development stages described in [7].

It has been established that an oscillating layer, or a layer of the maximum density gradient, can be formed during the surges. Its oscillations propagate into the overlying layers. The amplitude of such oscillations decreases with distance from the given layer, so that the liquid surface remains unperturbed. The quantitative characteristics of this vibrational motion are obtained: the amplitude of the oscillations reaches $1.2 \mathrm{~cm}$, the layer thickness is $1 \mathrm{~cm}$, the propagation velocity of the perturbations along the canal is $3-5 \mathrm{~cm} \cdot \mathrm{s}^{-1}$, and the oscillation period is $6 \mathrm{~s}$.

Presence of the bottom obstacle complicates the structure of the two-layer liquid motions. In particular, it results in emergence of the mixed layers and transformation of the flow behind the obstacle into a turbulent one, formation of the wave-like disturbances over the obstacle, sharp change of the interface position and occurrence of large-scale vortices with the horizontal axes. It is revealed that the maximum peak of the flow velocity horizontal component is shifted upstream from the obstacle. Comparison of the phenomena observed during flowing around under different wind conditions and different obstacle cross sections is given.

\section{REFERENCES}

1. Dobretsov, V.B., 1980. Osvoenie Mineral'nykh Resursov Shel'fa [Development of Mineral Shelf Resources]. Leningrad: Nedra, 272 p. (in Russian).

2. Bezrukov, Yu.F., 2001. Kolebaniya Urovnya i Volny v Mirovom Okeane [Level Fluctuations and Waves in the World Ocean]. Simferopol: V.I. Vernadsky TNU, 50 p. (in Russian).

3. Krauss, V., 1968. Vnutrennie Volny [Internal Waves]. Leningrad: Gidrometeoizdat, 272 p. (in Russian).

4. Helfrich, K.R. and Melville, W.K., 2006. Long Nonlinear Internal Waves. Annu. Rev. Fluid Mech., [e-journal] 38, pp. 395-425. doi:10.1146/annurev.fluid.38.050304.092129 
5. Shelkovnikov, N.K. and Alyavdin, G.I., 1982. Eksperimental'noe Issledovanie Protsessa Vovlecheniya v Dvukhsloynoy Zhidkosti [An Experimental Study of the Interface Dynamics in a Two-Layer Liquid]. Okeanologiya, 22(2), pp. 196-203 (in Russian).

6. Dotsenko, S.F. and Shelkovnikov, N.K., 2009. Laboratory Modeling of the Propagation of Periodic Internal Waves over Bottom Slopes. Physical Oceanography, [e-journal] 19(5), pp. 301-309. doi:10.1007/s11110-010-9055-5

7. Skorer, R., 1980. Aerogidrodinamika Okruzhayushchey Sredy [Environment Aerodynamics]. Moscow: Mir, pp. 250-269 (in Russian).

8. Liapidevskii, V.Yu., 2004. Mixing Layer on the Lee Side of an Obstacle. J. Appl. Mech. Tech. Phys., [e-journal] 45(2), pp. 199-203. doi:10.1023/B:JAMT.0000017582.70655.d9

9. Long, R.R., 1953. Some Aspects of the Flow of Stratified Fluids. 1. A Theoretical Investigation. Tellus, [e-journal] 5(1), pp. 42-58. doi:10.3402/tellusa.v5i1.8563 